\title{
Áreas potenciales para establecer Unidades Productoras de Germoplasma Forestal con dos variedades de Pinus pseudostrobus en México
}

\author{
Potential areas to establish Forest Germplasm Production Units \\ with two varieties of Pinus pseudostrobus in Mexico
}

\author{
Rubén Barrera Ramírez a, Rigoberto González Cubas a, Eduardo Javier Treviño Garza ${ }^{\text {a*}}$, \\ Benedicto Vargas Larreta ${ }^{\text {b }}$, Ricardo López Aguillón a \\ * Autor de correspondencia: a Universidad Autónoma de Nuevo León, Facultad de Ciencias Forestales, \\ Carretera Nacional km 145, s/n Linares, Nuevo León, México, C.P. 67700, tel.: (01) 8212140000 ext. 111, \\ eduardo.trevinogr@uanl.edu.mx \\ ${ }^{\mathrm{b}}$ Instituto Tecnológico de El Salto, El Salto, Pueblo Nuevo, Durango, México.
}

\begin{abstract}
SUMMARY
Areas with potential to establish Forest Germplasm Production Units (UPGF) with two varieties of P. pseudostrobus were determined. The objectives were (1) to model the current potential distribution of Pinus pseudostrobus var. pseudostrobus and P. pseudostrobus var. apulcensis using MaxEnt algorithms, and (2) to propose areas to locate the best individuals and establish UPGF in Michoacán and Oaxaca, Mexico. For these purposes, 2,451 data of presence obtained from own field records, Forest Biometric System, Global Biodiversity Information Facility and MEXU National Herbarium were used, as well as 19 predictor variables. The $75 \%$ of the records were used for training and $25 \%$ in validation. The adjustment and predictive capacity of ten models was evaluated; eight with 1,000 interactions and two with 500 were evaluated through precision tests of AUC (Area Under the Curve), ROC-standard (Receiver Operating Characteristics) and partial ROC. The models indicated that the two varieties present similar zones of potential distribution in a portion of the Neovolcanic Axis and Sierra Madre del Sur. The environmental variables with the most important contribution to the model for the pseudostrobus variety were those related to temperature; and for the apulcensis variety, temperature and precipitation. The establishment of UPGF can be carried out in areas of optimal growth; in addition, the data generated allow reducing the time and cost for the selection of the best individuals to be included in genetic improvement programs.
\end{abstract}

Key words: Optimal areas, potential distribution, Michoacan and Oaxaca, UPGF, varieties of Pinus pseudostrobus.

\section{RESUMEN}

Se determinaron áreas con potencial para establecer Unidades Productoras de Germoplasma Forestal (UPGF) con dos variedades de P. pseudostrobus. Los objetivos fueron (1) modelar la distribución potencial actual de Pinus pseudostrobus var. pseudostrobus y P. pseudostrobus var. apulcensis utilizando algoritmos de MaxEnt y (2) proponer áreas para localizar los mejores individuos y establecer UPGF en Michoacán y Oaxaca, México. Se utilizaron 2.451 datos de presencia obtenidos de registros propios en campo, Sistema Biométrico Forestal, Global Biodiversity Information Facility y del Herbario Nacional MEXU, así como 19 variables predictoras. El $75 \%$ de los registros se utilizaron para el entrenamiento y $25 \%$ en la validación. Se evaluó el ajuste y capacidad predictiva de diez modelos, ocho con 1.000 interacciones y dos con 500, a través de pruebas de precisión de AUC (Area Under the Curve), ROC-estándar (Receiver Operating Characteristics) y ROC-parcial. Los modelos indicaron que las dos variedades presentan zonas similares de distribución potencial en una porción del Eje Neovolcánico y la Sierra Madre del Sur, las variables ambientales con mayor contribución al modelo para la variedad pseudostrobus fueron las relacionadas con la temperatura, y para la variedad apulcensis temperatura y precipitación. El establecimiento de UPGF se puede realizar en zonas de crecimiento óptimo, además los datos generados permiten reducir el tiempo y costo para la selección de mejores individuos para ser incluidos en programas de mejoramiento genético.

Palabras clave: áreas óptimas, distribución potencial, Michoacán y Oaxaca, UPGF, variedades de P. pseudostrobus.

\section{INTRODUCCIÓN}

Las plantaciones forestales permiten solucionar problemas de baja productividad forestal, también permiten restaurar suelos forestales degradados. Un factor clave del éxito de las plantaciones es la producción y manejo de se- millas de calidad (Zobel y Talbert 1988). A nivel mundial, las especies del género Pinus son de las más utilizadas en el establecimiento de plantaciones forestales comerciales (PFC) debido a su alta rentabilidad económica y comercial, además de su capacidad de adaptación a distintas condiciones de sitio (FAO 2018). En México existen 49 de las 
120 especies reportadas para este género (Farjon y Styles 1997). Debido a la gran diversidad climática, topográfica y biológica del país es importante la elección de la especie y la variedad correcta para establecer PFC (Viveros Viveros et al. 2006). La selección adecuada del material genético recolectado aumenta la supervivencia y producción de las plantaciones (Flores et al. 2019). Sin embargo, los programas de plantaciones forestales comerciales en México utilizan fuentes de semillas en las que no se considera la calidad genética o el origen de los individuos (Castellanos Acuña et al. 2018). Desde su publicación en 2016, la Norma Mexicana NMXAA-169-SCFI-2016 (Secretaría de Economía 2016) especifica los requerimientos para el establecimiento y manejo de Unidades Productoras de Germoplasma Forestal (UPGF).

La selección de los mejores árboles es una actividad costosa y difícil de realizar (Reynoso et al. 2017), ya que los mayores costos se presentan durante su localización. Una manera de abatirlos es focalizar UPGF más pequeñas a través de diferentes herramientas de apoyo y planificación como los modelos de distribución potencial de especies. Dichos modelos son capaces de predecir la presencia de una especie en función de variables ambientales (Soberón 2010, Aguirre Gutiérrez et al. 2015) y se basan principalmente en correlaciones entre registros de presencia conocidos y sus condiciones ambientales específicas (Osorio Olvera et al. 2016). Para modelar la distribución de especies se han desarrollado diferentes técnicas basadas en algoritmos (Singh et al. 2018), siendo el modelo de máxima entropía (MaxEnt) el más aceptado (Radosavljevic y Anderson 2014). Este algoritmo estima la distribución de probabilidad de menor sesgo que minimiza la información, sujeta a un conjunto de restricciones basadas en el conocimiento de las condiciones ambientales en los sitios de presencia de una especie (Radosavljevic y Anderson 2014). También hace referencia al diagrama BAM, el cual esquematiza el área de distribución de la especie en determinado tiempo como resultado de una combinación de variables bióticas (B), ambientales (A) y el área de distribución que la especie ha podido alcanzar durante un período de tiempo relevante (M) (Soberón 2010).

En este estudio se aplicaron modelos de distribución potencial de especies para Pinus pseudostrobus Lindl. var. pseudostrobus y Pinus pseudostrobus var. apulcensis Martínez (sinonimia de P. pseudostrobus var. oaxacana Martínez; P. oaxacana Mirov). Estas variedades son utilizadas para el establecimiento de plantaciones forestales comerciales debido a su rentabilidad, la calidad de sus productos y su demanda comercial e industrialización (Perry 1991, Farjon y Styles 1997) en los estados de Michoacán y Oaxaca. Sin embargo, existen pocos trabajos relativos a la conservación y regularización del uso de su germoplasma, por lo que los objetivos son (1) modelar la distribución potencial actual de P. pseudostrobus. var pseudostrobus y P. pseudostrobus var. apulcensis y (2) proponer áreas para localizar los mejores individuos y establecer UPGF en dos de los estados de mayor producción forestal de la región centro-sur de México.

\section{MÉTODOS}

El estudio se llevó a cabo en bosques templados de los estados de Michoacán y Oaxaca, México, dos de las entidades con mayor producción forestal en la región centro y sur del país (figura 1).

Estas entidades se encuentran en una porción del Eje Neovolcánico y Sierra Madre del Sur donde la variedad pseudostrobus y variedad apulcensis crecen naturalmente (Farjon y Styles 1997). Los requerimientos agroecológicos de las variedades seleccionadas se obtuvieron del análisis de la información que se presenta en el Inventario Nacional Forestal y de Suelos (CONAFOR 2019), y del mapa de climas del Instituto Nacional de Estadística y Geografía (INEGI 2008) con el que se determinó la precipitación y temperatura promedio (cuadro 1).

Obtención de los datos de presencia. Los registros de presencia de cada una de las variedades fueron obtenidos en dos fases.

Fase 1: para obtener la distribución potencial actual de la especie, se incluyeron los registros de presencia en las Unidades de Manejo Forestal (UMAFOR) obtenidos del inventario del Sistema Biométrico Forestal para el manejo de bosques templados y selvas de México (SiBiFor) para los estados de Michoacán y Oaxaca. Para complementar la base de datos, también se obtuvieron registros del Herbario Nacional (MEXU) del Instituto de Biología de la Universidad Nacional Autónoma de México (UNAM 2019) y del portal Global Biodiversity Information Facility (GBIF 2019) que incluye datos de herbarios de todo el mundo (periodo no mayor a 50 años).

Fase 2: para determinar las áreas con potencial óptimo de selección de los mejores individuos, y para establecer UPGF, se utilizaron datos de registros propios en campo obtenidos de 2014 a 2018 que corresponden a árboles superiores (árbol con características fenotípicas deseables) seleccionados a través del método de comparación entre vecinos, propuesto por Zobel y Talbert (1988). Este método consiste en elegir un árbol candidato a superior para compararlo cualitativa y cuantitativamente con árboles testigos (tres a cinco árboles de la misma especie que crecen en las mismas condiciones de sitio) y que finalmente son dictaminados como árboles superiores. Se obtuvieron 2.217 registros georreferenciados en campo (2.100 para P. pseudostrobus var. pseudostrobus y 117 de la var. apulcensis), y de las fuentes consultadas (MEXU y GBIF) 234 registros (154 para $P$. pseudostrobus var. pseudostrobus y 80 de $P$. pseudostrobus var. apulcensis); en total se incluyeron 2.451 registros.

Los registros se depuraron en varias fases antes de iniciar la modelación. Primero, se eliminaron datos repetidos, 
mal georreferenciados y cuya identidad taxonómica hasta especie estaba en duda. Posteriormente, con el programa NicheToolBox de la Comisión Nacional para el Conocimiento y Uso de la Biodiversidad (Osorio Olvera et al. 2016), se excluyeron todos los valores atípicos geográficos y atípicos climáticos, es decir, donde se reportó la especie, pero se encontró fuera de su distribución natural. Finalmente, para evitar problemas de "sobremodelación" los puntos de presencia se depuraron y se dejó solo un registro por celda $\left(1 \mathrm{~km}^{2}\right)$.
Variables predictoras. La construcción del modelo se realizó con 19 variables bioclimáticas descargadas de la plataforma del Centro de Recursos Idrisi-México de la Universidad Autónoma del Estado de México (CRI-UAEMéx) (Cuervo Robayo et al. 2014), las cuales representan el comportamiento climático promedio en México para los años 1910-2009, con resolución espacial de 30 segundos de arco $\left(1 \mathrm{~km}^{2}\right)$. Para evitar sobreajuste del modelo (Peterson et al. 2011), se realizó un análisis de correlación de Pearson entre cada uno de los valores puntuales de las 19

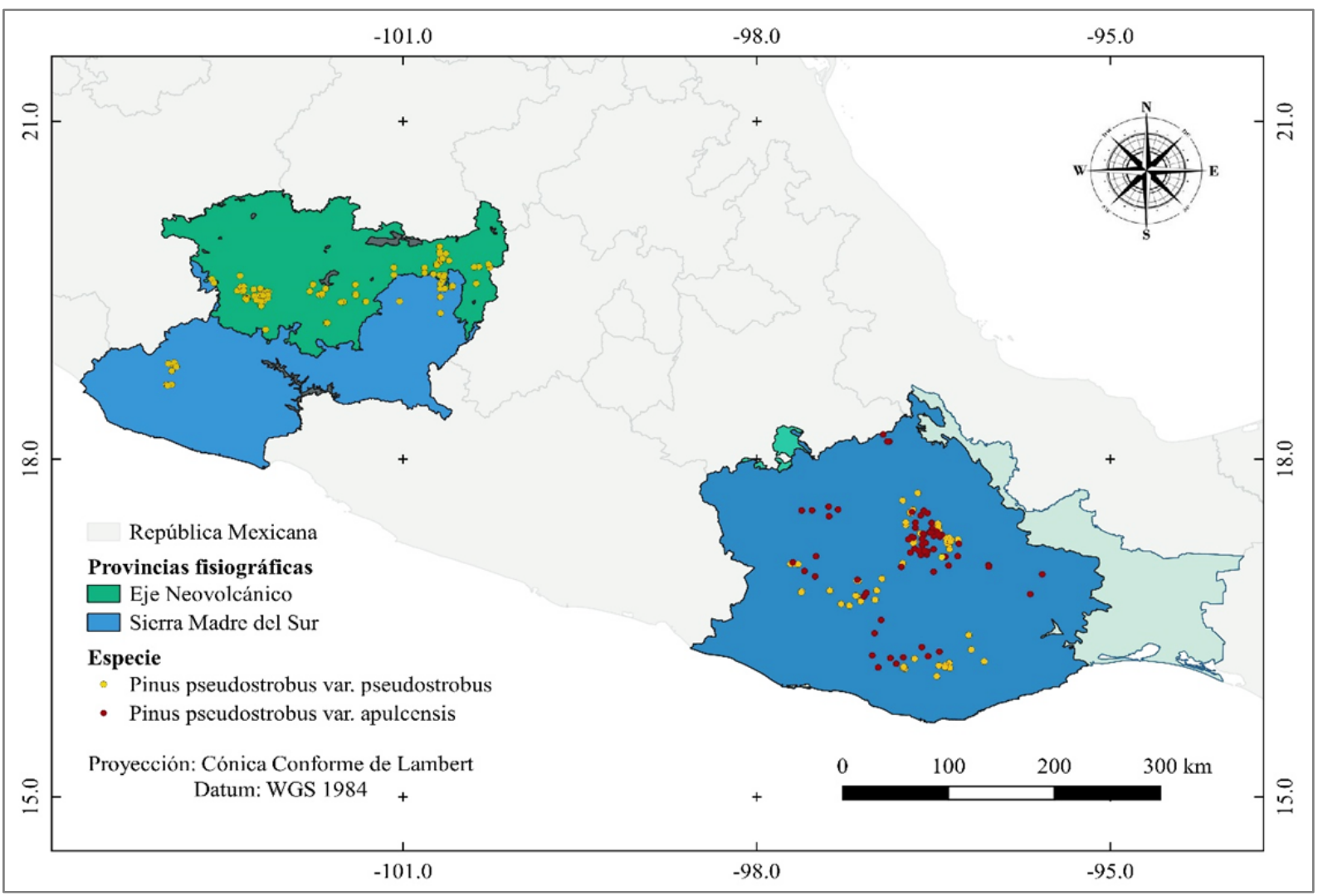

Figura 1. Distribución natural de Pinus pseudostrobus var. pseudostrobus y P. pseudostrobus var. apulcensis en Michoacán y Oaxaca, México. Fuente: Elaboración propia con datos de INEGI, registros propios y del Sistema Biométrico Forestal.

Natural distribution of Pinus pseudostrobus var. pseudostrobus and P. pseudostrobus var. apulcensis in Michoacan and Oaxaca, Mexico. Source: Own elaboration with data from INEGI, own records and from Forest Biometric System.

Cuadro 1. Requerimientos agroecológicos de Pinus pseudostrobus var. pseudostrobus y P. pseudostrobus var. apulcensis.

Agroecological requirements of Pinus pseudostrobus var. pseudostrobus and P. pseudostrobus var. apulcensis.

\begin{tabular}{lcccccc}
\hline Especie & $\begin{array}{c}\text { Altitud } \\
(\mathrm{m} \mathrm{snm})\end{array}$ & $\begin{array}{c}\text { Precipitación anual } \\
\text { total }(\mathrm{mm})\end{array}$ & $\begin{array}{c}\text { Temperatura } \\
\text { media anual }\left({ }^{\circ} \mathrm{C}\right)\end{array}$ & $\begin{array}{c}\text { pH del } \\
\text { suelo }\end{array}$ & $\begin{array}{c}\text { Textura del } \\
\text { suelo }(\text { Clase })\end{array}$ & $\begin{array}{c}\text { Profundidad } \\
\text { del suelo }(\mathrm{m})\end{array}$ \\
\hline $\begin{array}{l}\text { P.pseudostrobus var. } \\
\text { pseudostrobus }\end{array}$ & 1.650 a 3.300 & 1.000 a 1.400 & $12-18$ & 5 a 6,5 & Gruesa / media & 1 a 3 \\
\hline $\begin{array}{l}\text { P.pseudostrobus var. } \\
\text { apulcensis }\end{array}$ & 1.400 a 3.200 & 800 a 1.400 & $12-20$ & 4,5 a 7 & Gruesa / media & 0,5 a 3 \\
\hline
\end{tabular}


capas bioclimáticas. Se eliminaron las variables que mostraron alta multicolinealidad (debido a que presentan relaciones aproximadamente lineales entre los regresores del modelo, cuando los estimadores obtenidos y la precisión de estos se ven seriamente afectados) y se conservaron solo aquellas variables que maximizan la contribución al modelo; el análisis se llevó a cabo mediante el programa Statistica ver. 13.0.

Además, se incluyeron variables del sitio como: pendiente $(\%)$, exposición $\left({ }^{\circ}\right)$ y altitud $(\mathrm{m})$ obtenidas del Modelo Digital de Elevación del Instituto Nacional de Estadística y Geografía (INEGI 2019). Para que los mapas coincidieran con las fuentes de Worldclim (Worldclim 2019), en los complementos de GdalTools de la biblioteca de Qgis 3.2.3 (QGIS 2019) con la herramienta de "alineación de ráster" se estableció una sola resolución $\left(1 \mathrm{~km}^{2}\right)$. Para generar el modelo se utilizaron 16 variables bioclimáticas y tres variables de sitio (cuadro 2).

Delimitación del área accesible (símbolo M). El concepto de área accesible para una especie (M) hace referencia al diagrama BAM (Soberón 2010). Para delimitar el área accesible (M), se utilizaron las Provincias Fisiográficas de México y se escogieron aquellas que coincidieron con los registros de presencia para cada variedad, evitando provincias que, aunque similares, no tuvieron registros. A partir de la delimitación del área $\mathrm{M}$ se recortaron las capas ambientales para cada variedad en formato ASCII con el uso del programa Qgis 3.2.3 (QGIS 2019).

Generación del modelo. El análisis del área potencial para el establecimiento de UPGF se realizó con el algoritmo de máxima entropía del programa MaxEnt ver. 3.4.1, el cual permite escoger los modelos más consistentes a partir de información disponible de datos de presencia; no requiere datos de ausencia y genera buenas predicciones, aún con muestras pequeñas (Radosavljevic y Anderson 2014).

Se utilizaron los parámetros de elaboración que vienen por defecto en el programa, a excepción de las opciones Extrapolate y Do clamping que se desactivaron para evitar extrapolaciones artificiales en los valores extremos de las variables bioclimáticas. Se obtuvo una salida tipo logística

Cuadro 2. Variables incorporadas en la modelación de la distribución potencial de Pinus pseudostrobus var. pseudostrobus y P. pseudostrobus var. apulcensis y para la selección de Unidades Productoras de Germoplasma Forestal en Oaxaca y Michoacán ( $\mathrm{r} \geq 0,85$ ).

Variables incorporated in the modeling of the potential distribution of Pinus pseudostrobus var. pseudostrobus and P. pseudostrobus var. apulcensis and for the selection of Forest Germplasm Production Units in Oaxaca and Michoacán ( $\mathrm{r} \geq 0.85)$.

\begin{tabular}{|c|c|}
\hline Código & Descripción de las variables bioclimáticas (unidad) \\
\hline Biol & Temperatura promedio anual $\left({ }^{\circ} \mathrm{C}\right)$ \\
\hline Bio2 & Oscilación diurna de la temperatura $\left({ }^{\circ} \mathrm{C}\right)$ \\
\hline Bio3 & Isotermalidad $\left({ }^{\circ} \mathrm{C}\right)$ \\
\hline Bio4 & Estacionalidad de la temperatura (desviación estándar *100) $\left({ }^{\circ} \mathrm{C}\right)$ \\
\hline Bio5 & Temperatura máxima promedio del periodo más cálido $\left({ }^{\circ} \mathrm{C}\right)$ \\
\hline Bio6 & Temperatura mínima del mes más frío $\left({ }^{\circ} \mathrm{C}\right)$ \\
\hline Bio7 & Oscilación anual de la temperatura $\left({ }^{\circ} \mathrm{C}\right)$ \\
\hline Bio8 & Temperatura media del mes más húmedo $\left({ }^{\circ} \mathrm{C}\right)$ \\
\hline Bio9 & Temperatura media del mes más seco $\left({ }^{\circ} \mathrm{C}\right)$ \\
\hline Bio10 & Temperatura media del cuatrimestre más cálido $\left({ }^{\circ} \mathrm{C}\right)$ \\
\hline Bio14 & Precipitación del periodo más seco $(\mathrm{mm})$ \\
\hline Bio15 & Estacionalidad de la precipitación (Coeficiente de variación, CV) \\
\hline Bio16 & Precipitación del trimestre más húmedo $(\mathrm{mm})$ \\
\hline Bio17 & Precipitación del trimestre más seco $(\mathrm{mm})$ \\
\hline Bio18 & Precipitación del cuatrimestre más cálido (mm) \\
\hline Bio19 & Precipitación del cuatrimestre más frío (mm) \\
\hline \multicolumn{2}{|r|}{ Variables del sitio } \\
\hline Exp & Exposición $\left(^{\circ}\right)$ \\
\hline Alt & Altitud (m) \\
\hline Pdt & Pendiente $(\%)$ \\
\hline
\end{tabular}

Fuente: Worldclim ver 2.1, (2019) e INEGI (2019). 
usando un umbral 0-1 ( $0=$ ausencia y $1=$ presencia $)$, los valores cercanos a $0(<0,5)$ indican que las condiciones no son idóneas y valores superiores a 0,5 que las condiciones son adecuadas (Cuervo Robayo et al. 2014).

El $75 \%$ de los registros se utilizó para el entrenamiento y $25 \%$ para la validación; se probaron diez modelos por variedad, ocho con 1.000 interacciones y dos con 500 (valor por defecto-Bootstrap) y límite de convergencia fijo de 0,00001 (valor por omisión), mediante dos procedimientos: (1) por su desempeño y ajuste estadístico en las pruebas de AUC (Area Under the Curve) y del análisis de precisión ROC (Receiver Operating Characteristic) para determinar la validez de los modelos y una prueba $\mathrm{Z}$ que determina si dicha relación es menor o cercana a 1 (Radosavljevic y Anderson 2014); (2) mediante salidas a campo para realizar la evaluación de la distribución de ambas variedades de $P$. pseudostrobus, registrando la presencia o ausencia en el área del modelo.

Validación del modelo. Los modelos con valores de AUC entre 0,7-0,9 para los puntos de entrenamiento y de prueba se consideraron buenos y los modelos con valores $\geq 0,9$ como excelentes (Peterson et al. 2011). El análisis de significancia estadística de la predicción se realizó a través de 10 pruebas binomiales de omisión al $99 \%$ de confiabilidad. Sin embargo, dado que la utilidad de los análisis ROC ha sido cuestionada por especialistas debido a que los algoritmos solo utilizan datos de presencia y en teoría, también requiere datos de ausencia verdadera (Martínez Méndez et al. 2016), se realizó el análisis ROC-parcial con el programa Tool for Partial-ROC que permitió contrarrestar las diferencias de AUC. Las corridas se hicieron para cada modelo, utilizando $50 \%$ de los puntos de evaluación independientes remuestreados en 1.000 réplicas bootstrap y un error fijo de omisión no mayor a $5 \%$ (1-omission threshold $>0,95)$. El mejor modelo se eligió de acuerdo con el valor más alto de AUC; valores $>1$ indican que las predicciones se deben a la modelación y no al azar (Soberón 2010, Peterson et al. 2011). Finalmente, de acuerdo con Martínez Méndez et al. (2016) se realizó una prueba $\mathrm{Z}$ para determinar si los valores de las proporciones AUC parciales de los modelos de idoneidad eran estadísticamente mejores que un modelo al azar $(\mathrm{AUC}=1,0)$.

Distribución potencial actual. Se utilizó la prueba Jackknife para conocer el porcentaje de contribución de cada variable al modelo de distribución actual, con los resultados se identificaron áreas de distribución potencial para ambas variedades. Además, se realizaron recorridos de campo con el propósito de validar los resultados de la modelación, lo que permitió garantizar que efectivamente existen árboles con características fenotípicas deseables. Con la información obtenida del modelo se reclasificaron valores del archivo ráster en tres categorías de probabilidad de ocurrencia: baja $(0,6-0,7)$, media $(0,7-0,8)$ y alta $(0,8-1,0)$ y se calculó la superficie potencial actual por decil.
Áreas óptimas para el establecimiento de UPGF y selección de los mejores individuos. Para identificar zonas con potencial óptimo para el establecimiento de UPGF, se calculó la superficie potencial a partir de la reclasificación en categorías binarias de 0 (área no apta) y 1 (área con potencial óptimo) del mejor modelo y se calculó la superficie por decil para conocer el área potencial óptima correspondiente a Oaxaca y Michoacán. Las proyecciones de los modelos y la creación de mapas se realizaron con el programa Qgis 3.2.3 (QGIS 2019).

\section{RESULTADOS}

Distribución potencial actual. Después del proceso de depuración de la base de datos el número final de registros fue de 494 para P. pseudostrobus var. pseudostrobus y 143 para $P$. pseudostrobus var. apulcensis. La mayoría de los datos eliminados fueron de registros repetidos en un mismo píxel $\left(1 \mathrm{~km}^{2}\right)$ o mal georreferenciados. Para delimitar el área óptima de crecimiento y distribución de la especie (M) se utilizaron dos provincias fisiográficas. La variedad pseudostrobus se localizó en el Eje Neovolcánico en el estado de Michoacán y la Sierra Madre del Sur en el estado de Oaxaca. Por su parte, la variedad apulcensis solo se localizó en esta última. El área potencial fue similar en más del $50 \%$ de los casos en ambas provincias fisiográficas; de hecho, en el estado de Oaxaca las dos variedades crecen juntas en la mayoría de los sitios.

De acuerdo con la prueba de ROC estándar, los 10 modelos de idoneidad probados para ambas variedades fueron estadísticamente mejores que el modelo al azar según la prueba binomial, que en todos los casos mostró valores significativos $(P<0,01)$. Los resultados de las pruebas de ROC parcial arrojaron razones de AUC con valores significativos $(P<0,0001)$ mayores a 1 . Por lo tanto, los modelos se catalogaron como muy buenos. Los valores del AUC para los datos de entrenamiento de la variedad pseudostrobus fueron de 0,968 a 0,969 y de 0,963 a 0,970 para la validación. Para la variedad apulcensis los valores de AUC para el entrenamiento fueron de 0,959 a 0,969 y para la validación de 0,942 a 0,972 (cuadro 3 ).

Los resultados de la prueba $\mathrm{Z}$ indicaron que todos los modelos fueron estadísticamente significativos $(P<0,01)$; sin embargo, para la variedad pseudostrobus, el modelo dos (M2) presentó el mejor ajuste, con un valor de AUC de 0,969 para el entrenamiento, de 0,964 para la validación y 1,385 para ROC parcial. Para la variedad apulcensis el modelo siete (M7) presentó los mejores valores de AUC, con 0,963 para el entrenamiento, 0,972 en la validación y 1,174 en la prueba ROC parcial (cuadro 3).

Ambas variedades presentan zonas similares de distribución potencial (potencial alto: color verde, figura 2), en rangos altitudinales óptimos de 2.200 a 3.200 m (2.700 m en promedio) característicos de bosques templados de coníferas. En el estado de Michoacán, la superficie con distribución potencial alta para la variedad pseudostrobus es de 
Cuadro 3. Valores de las proporciones AUC del análisis de ROC parcial y de Z para los dos mejores modelos de idoneidad en Pinus pseudostrobus var. pseudostrobus y P. pseudostrobus var. apulcensis.

Values of the AUC proportions of the partial ROC and Z analysis for the two best suitability models in Pinus pseudostrobus var. pseudostrobus and P. pseudostrobus var. apulcensis.

\begin{tabular}{lccccc}
\hline \multicolumn{1}{c}{ Variedad } & Modelo & $\begin{array}{c}\text { Valor de AUC } \\
\text { en la validación }\end{array}$ & $\begin{array}{c}\text { Proporciones AUC } \\
\text { del ROC parcial }\end{array}$ & $\begin{array}{c}\text { DE } \\
\text { Prueba Z } \\
\text { valor } P\end{array}$ \\
\hline $\begin{array}{l}\text { P. pseudostrobus var. pseudostrobus } \\
\text { P. pseudostrobus var. apulcensis }\end{array}$ & M2 & $0,964^{*}$ & 1,385 & 0,003 & $P<0,01$ \\
\hline
\end{tabular}

M2: Modelo dos, M7: Modelo siete, * Significativos con $P<0,01$, DE: Desviación estándar.

Cuadro 4. Contribución porcentual de las variables a los modelos generados para Pinus pseudostrobus var. pseudostrobus y P. pseudostrobus var. apulcensis de Oaxaca y Michoacán.

Percentage contribution of the variables to the models generated for Pinus pseudostrobus var. pseudostrobus and P. pseudostrobus var. apulsencis from Oaxaca and Michoacan.

\begin{tabular}{lclc}
\hline \multicolumn{2}{c}{$\begin{array}{c}\text { P. pseudostrobus var. } \\
\text { pseudostrobus }\end{array}$} & \multicolumn{2}{c}{$\begin{array}{c}\text { P.peudostrobus var. } \\
\text { apulcensis }\end{array}$} \\
\hline Variable & $\begin{array}{c}\text { Contribución } \\
\text { porcentual }\end{array}$ & Variable & $\begin{array}{c}\text { Contribución } \\
\text { porcentual }\end{array}$ \\
\hline Bio4 & 48,1 & Bio8 & 46,0 \\
Bio10 & 29,3 & Bio19 & 23,9 \\
Bio2 & 6,6 & Bio14 & 4,2 \\
Bio1 & 4,6 & Bio9 & 3,9 \\
Bio5 & 3,7 & Bio18 & 3,7 \\
Bio16 & 2,0 & Bio6 & 3,5 \\
Bio3 & 1,3 & Bio3 & 2,9 \\
Bio19 & 0,9 & Bio1 & 2,6 \\
Bio8 & 0,8 & Bio10 & 2,3 \\
Bio9 & 0,7 & Bio15 & 2,0 \\
Bio7 & 0,5 & Bio07 & 1,2 \\
Bio6 & 0,4 & Bio16 & 1,1 \\
Bio17 & 0,4 & Bio04 & 0,9 \\
Bio14 & 0,3 & Bio05 & 0,9 \\
Bio18 & 0,2 & Bio02 & 0,8 \\
Bio15 & 0,2 & - & - \\
\hline & & & \\
\hline
\end{tabular}

152.700 ha, media de 721.600 ha y baja de 787.800 ha. En Oaxaca existen 106.200 ha de distribución potencial alta, media de 1.660.200 ha y baja de 1.811 .800 ha, el área de traslape de la distribución potencial equivale al 73,3 \% del área total, por lo tanto, existen 2.620 .316 ha donde potencialmente ambas variedades pueden encontrarse interactuando, esto indica que las dos variedades tienen un nicho similar (espacio ambiental), pero que no es equivalente o idéntico en su totalidad (figura 2).
Las variables de mayor contribución al modelo para la variedad pseudostrobus fueron: Bio4 (Estacionalidad de la temperatura que expresa la desviación estándar de la temperatura media mensual * 100, en cada estación: $48,1 \%$ ), Bio10 (temperatura media del cuatrimestre más cálido: $29,3 \%$ ) y Bio2 (oscilación diurna de la temperatura: 6,6 \%); en contraste, para la variedad apulcensis, fueron Bio8 (temperatura media del mes más húmedo: $46 \%$ ), Bio19 (precipitación del cuatrimestre más frío: 23,9\%) y Bio14 (precipitación del periodo más seco: 4,2\%). Ambas variedades presentaron en común algunas variables, pero con distinta proporción (cuadro 4).

Áreas óptimas para el establecimiento de UPGF y selección de los mejores individuos. Ambas variedades tienen zonas de distribución potencial similares, por ejemplo, la variedad pseudostrobus se desarrolla principalmente de 2.200 a 2.800 m s.n.m., con excepciones desde 1.650 a 2.200 m s.n.m. y más de 2.800 a 3.400 m s.n.m. Por su parte, la variedad apulcensis se desarrolla óptimamente de 2.400 a $2.850 \mathrm{~m}$ s.n.m., con excepciones de 1.400 a 2.400 m s.n.m. y más 2.850 a 3.300 m s.n.m. La mejor condición con potencial óptimo $=1$ para la variedad pseudostrobus y el establecimiento de UPGF en Michoacán se presentó en el Eje Neovolcánico donde más del $50 \%$ del área se considera como óptima; mientras que en Oaxaca las mejores condiciones para ambas variedades ocurren en la Sierra Madre del Sur ya que más del 70 \% del área es idónea para el establecimiento de UPGF.

De acuerdo con el modelo generado, al categorizar las áreas de idoneidad en óptima (1) y no óptima (0) para el establecimiento de UPGFs con la variedad pseudostrobus en el estado de Michoacán se obtuvo una superficie óptima de 938.000 ha que equivale al $42,6 \%$ de su territorio forestal (2,2 millones de ha). En Oaxaca existen 989.300 ha con potencial óptimo para establecer UPGF con ambas variedades, que representan el $14 \%$ del total de la superficie forestal del estado (7,06 millones de ha) (figura 3). Con los datos del modelo y los recorridos de campo permitieron registrar dos UPGF (que corresponden a árboles superiores ya seleccionados; figura 3), una para el estado de Michoacán con P. pseudostrobus var. pseudostrobus localizados dentro de la provincia Eje Neovolcánico, y la otra para Oa- 


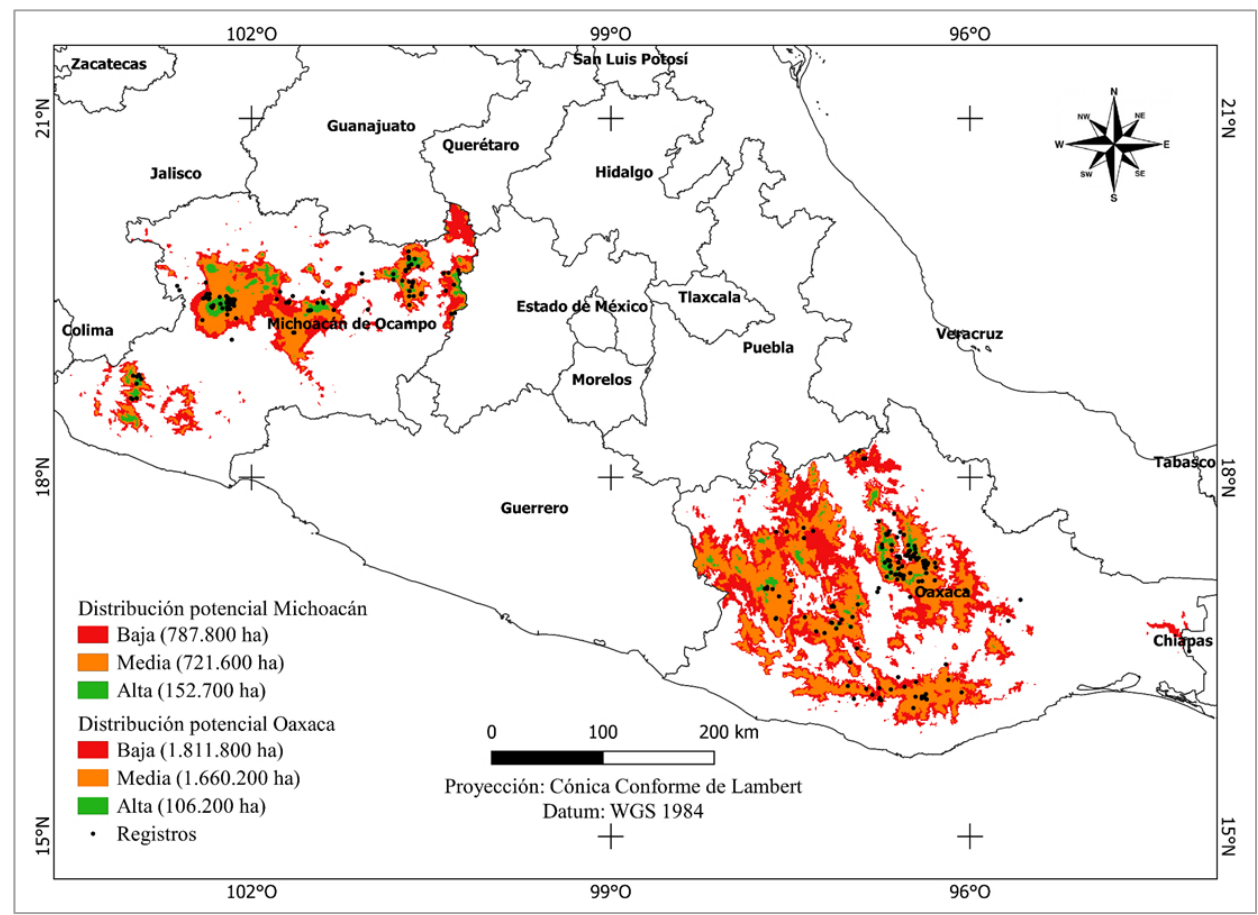

Figura 2. Distribución potencial de Pinus pseudostrobus var. pseudostrobus y P. pseudostrobus var. apulcensis en una porción del Eje Neovolcánico y de la Sierra Madre del Sur en Michoacán y Oaxaca, respectivamente.

Potential distribution of Pinus pseudostrobus var. pseudostrobus and P. pseudostrobus var. apulcensis, in a portion of Neovolcanic Axis and Sierra Madre del Sur in Michoacan and Oaxaca, respectively.

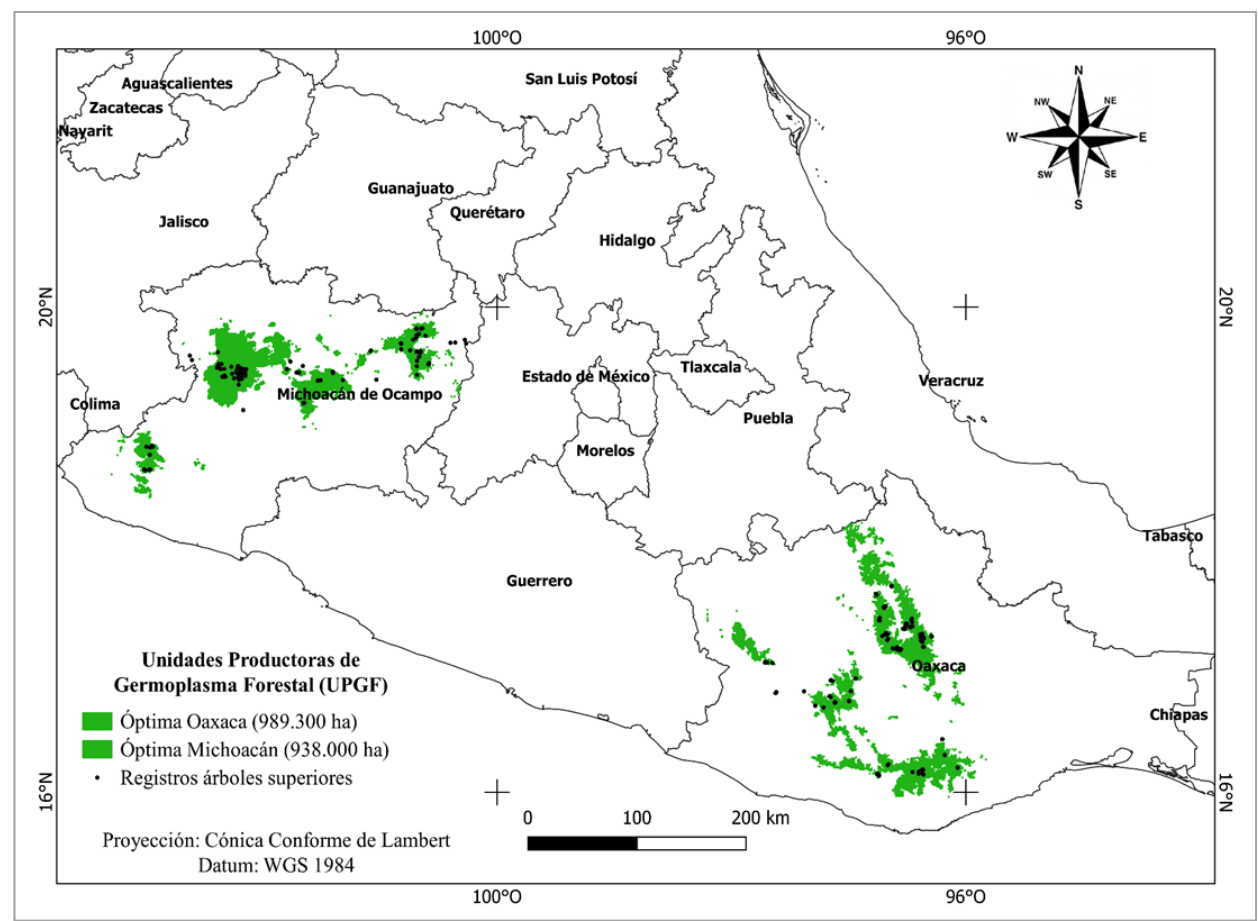

Figura. 3. Áreas con potencial óptimo para el establecimiento de UPGF con Pinus pseudostrobus var. pseudostrobus y P. pseudostrobus var. apulcensis. apulcensis.

Areas with optimal potential for the establishment of UPGF to Pinus pseudostrobus var. pseudostrobus and P. pseudostrobus var. 
xaca con ambas variedades ubicadas en distintas comunidades de la Sierra Juárez de Oaxaca, dentro de la provincia Sierra Madre del Sur. En ambos casos, de acuerdo con los resultados del modelo, el área donde se ubicaron las UPGF fue catalogada como óptima y con potencial alto para seleccionar en el futuro otros individuos con características sobresalientes o para establecer otras UPGF.

\section{DISCUSIÓN}

Distribución potencial actual. Los modelos obtenidos presentaron el mayor ajuste de acuerdo con los resultados de las pruebas de ROC estándar y ROC parcial, ya que estadísticamente fueron mejores que una modelación al azar (valores > 1) (Soberón 2010, García Aranda et al. 2018, Singh et al. 2018). El hecho de catalogar a los modelos como buenos se debe a que se usaron todos los datos de presencia en toda el área que ocupan las dos variedades para Oaxaca y Michoacán, junto con los datos de inventarios y registros propios en campo (caso de árboles superiores), que fueron delimitados con las provincias fisiográficas de México (Eje Neovolcánico y Sierra Madre del Sur), para que con mayor precisión se estableciera la distribución potencial actual (Cuervo Robayo et al. 2014); es decir, el área $\mathrm{M}$ óptima de acuerdo al diagrama BAM (Soberón 2010). Con los resultados obtenidos se puede aseverar que ambas variedades en el estado de Oaxaca ocupan un área de idoneidad similar, ya que se encuentran coexistiendo en algunos sitios de bosques templados de la Sierra Madre del Sur. En Michoacán, la variedad pseudostrobus se localizó en bosques templados del Eje Neovolcánico; en ambos casos, estos resultados coinciden con los conocimientos básicos de la biología de ambas variedades (Farjon y Styles 1997).

Los estudios que existen sobre la modelación de idoneidad de hábitat para especies del mismo género se centran principalmente en la teoría de nicho ecológico (Reynoso et al. 2017, García Aranda et al. 2018, ManzanillaQuiñones et al. 2019, Flores et al. 2019), utilizando en su mayoría datos de centros de recolección de muestras botánicas, por ejemplo del Herbario Nacional-MEXU (UNAM 2019), o de plataformas digitales como Global Biodiversity Information Facility (GBIF 2019) que solo consideran la presencia de la especie. Sin embargo, si no se realiza una correcta depuración de estos datos (registros mayores de 50 años) puede haber errores con datos repetidos y mal georreferenciados, debido a que el intervalo real de tolerancia climática (intervalo óptimo) es variable entre especies o entre variedades, y aunque convivan en la misma región es posible que haya sobreestimación de los modelos (Perret et al. 2019). Por lo que es conveniente reforzar los registros obtenidos de plataformas digitales o de herbarios con datos de inventarios propios que hayan sido corroborados (como se hizo en este caso), como medida preventiva y de poco riesgo para obtener modelos más confiables.
En trabajos similares como el realizado por Reynoso et al. (2017) en Chiapas, Méx., utilizaron registros de $P$. pseudostrobus Lindl. y P. oocarpa Schiede ex Schltdl. procedentes de plataformas digitales y para modelar la superficie con potencial y establecer UPGF, emplearon variables bioclimáticas y edafoclimáticas (como Bio2, Altitud, Edafología, pH y textura, caso P. pseudostrobus y Bio2, Bio14, Altitud, Índice de vegetación diferencial normalizado y precipitación media anual para $P$. oocarpa); y obtuvieron que la mayor superficie con idoneidad para establecer UPGF con ambas especies se encontró al igual que en este trabajo, en una porción de la Sierra Madre del Sur. De manera similar, Cruz Cárdenas et al. (2016) modelaron la distribución potencial de 12 especies (dos del género Abies y 10 de Pinus, incluida la variedad pseudostrobus) en el estado de Michoacán, empleando cuatro variables de clima (Bio5, Bio6, Bio7 y Bio17), dos de suelo (pH y potasio) y el modelo digital de elevación de INEGI; donde las provincias fisiográficas con la mayor distribución potencial fueron el Eje Neovolcánico y la Sierra Madre del Sur, aunque, los autores no lograron separar las diferencias ambientales entre las especies, siendo las mismas condiciones ambientales idóneas para todas estas. Al igual que en este trabajo, se debe contemplar el área $\mathrm{M}$ de una especie y eliminar la autocorrelación de las variables del modelado, para evitar errores en la distribución potencial de la especie o variedad. En otro estudio más especializado realizado por Aguirre Gutiérrez et al. (2015) se comparó el nicho ecológico de cinco pinos del subgénero strobus, se emplearon variables bioclimáticas de Worldclim (resolución de $1 \mathrm{~km}^{2}$ ), edafoclimáticas, la radiación solar y el índice de vegetación de diferencia normalizada. Los autores señalaron que las áreas con mayor potencial y con alta idoneidad para estas especies se encontraron localizadas en el Eje Neovolcánico, la Sierra Madre del Sur y las Sierras de Chiapas y Guatemala, logrando encontrar diferencias ambientales entre las especies, donde las variables de mayor contribución al modelo fueron Bio3 ( $P$. ayacahuite Ehrenb. ex Schltdl y P. chiapensis (Martínez) Andresen), Bio15 (P. ayacahuite var. veitchii Roezl (Shaw), elevación (P. strobiformis Engelmann) y radiación solar (P. lambertiana D. Douglas). Los resultados de estos estudios refuerzan los obtenidos en el presente trabajo, ya que ambas variedades se localizan en dos centros de idoneidad ambiental en México (Eje Neovolcánico y la Sierra Madre del Sur) en convivencia con más especies del mismo género.

Las variables que mejor predicen la distribución potencial del género Pinus, sobre todo con especies de distribución amplia, se relacionan con la temperatura (García Aranda et al. 2018). Lo que coincide con los resultados obtenidos, ya que la variable con mayor contribución al modelo para la variedad pseudostrobus fue la estacionalidad de la temperatura (Bio4: 48,1 \%) y para la variedad apulcensis, la temperatura media del mes más húmedo (Bio8: 40,0 \%). Para especies de distribución restringida, la altitud influye más que la temperatura (Cruz Cárdenas 
et al. 2016, García Aranda et al. 2018). En otros estudios para especies de pino en México los resultados coinciden, ya que los modelos que incluyen variables de temperatura han contribuido mayormente para determinar el área potencial de especies con distribución amplia. Además, dichas especies coinciden con lo reportado en este estudio, ya que su área de idoneidad se ha localizado principalmente en el Eje Neovolcánico y la Sierra Madre del Sur (Aguirre Gutiérrez et al. 2015, Cruz Cárdenas et al. 2016, Reynoso et al. 2017, Manzanilla-Quiñones et al. 2019). En general, las variaciones en el ajuste del modelo y de la distribución potencial de la especie dependen del tipo de variable bioclimática, la escala y el número de registros de la especie modelada (García Aranda et al. 2018).

Áreas óptimas para el establecimiento de UPGF y selección de los mejores individuos. Un programa de mejora genética debe iniciar con la selección de los mejores individuos con base en su fenotipo (Zobel y Talbert 1988), y se debe enfocar en el aumento y calidad de los árboles y su adaptabilidad a cambios ambientales. Con los resultados obtenidos se puede realizar la planeación del establecimiento de huertos semilleros asexuales con árboles de las zonas de crecimiento óptimo para ambas variedades, como posible estrategia para generar UPGF, pero de mejor calidad genética, que a su vez permitan la conservación in situ de ambas variedades. El Eje Neovolcánico y la Serra Madre del Sur han sido reportadas como zonas de idoneidad para la distribución de varias especies de Pinus y Abies (incluida la var. pseudostrobus) (Aguirre Gutiérrez et al. 2015, Cruz Cárdenas et al. 2016, Martínez Méndez et al. 2016). La conservación de pinos debe considerar la protección de los rodales forestales actuales, y desarrollar estrategias para el establecimiento de nuevas UPGF que permitan su reintroducción en áreas deforestadas y/o perturbadas. Además, es importante considerar los efectos de la fragmentación del hábitat y el cambio de uso del suelo (Mantyka-Pringle et al. 2012), ya que a menudo ocurre en especies o variedades con distribución amplia (como en este caso) y que se encuentra fuera de áreas sujetas a protección.

Pinus pseudostrobus var. pseudostrobus y P. pseudostrobus var. apulcensis no se encuentran en alguna categoría de riesgo. Sin embargo, el aprovechamiento excesivo y disgénico, el cambio de uso de suelo y el aislamiento de poblaciones locales dan lugar a la fragmentación de hábitats, y como consecuencia la pérdida probable de rodales y árboles superiores (Aguirre Gutiérrez et al. 2015). De acuerdo con la Norma Mexicana NMXAA-169SCFI-2016 (Secretaría de Economía 2016), se requiere de acciones como el establecimiento de UPGF que tengan como objetivo la conservación de los mejores individuos, de importancia ecológica y económica.

Los datos generados en este trabajo son los primeros que describen áreas óptimas para incursionar en la selección de individuos sobresalientes con dos variedades de
P. pseudostrobus en el centro-sur de México donde ambas variedades tienen gran importancia económica y ecológica debido a su distribución, y también a que son utilizadas en la producción de madera aserrada de buena calidad, durmientes, tableros de partículas, madera de construcción, madera para chapa y triplay, celulosa y resina (Farjon y Styles 1997). Se han reportado diferencias de crecimiento en altura y diámetro entre variedades de este taxon (Viveros Viveros et al. 2006), por lo que debe cuidarse su correcto uso masivo, por ejemplo, en plantaciones forestales. Además, es conveniente explorar otras áreas de idoneidad y establecer UPGF con el objetivo de mejora genética para los estados de Michoacán y Oaxaca. Aunque los resultados obtenidos no se enfocan en el análisis del efecto del cambio climático sobre la subsistencia de estas variedades de pino, se puede emplear como base para compensar dichos cambios (Castellanos Acuña et al. 2018) y servir como fuentes de germoplasma en hábitats que en la actualidad son de mayor altitud (var. pseudostrobus) o de mayor humedad (var. apulcensis). Es decir, los resultados de este estudio pueden funcionar en escenarios climáticos futuros (probablemente más cálidos y secos) como base en actividades de migración asistida con fines de conservación o restauración de las áreas boscosas de estas variedades tal y como lo señalan Sáenz Romero et al. (2009) y Castellanos Acuña et al. (2018).

\section{CONCLUSIONES}

Las variables bioclimáticas que mayor influencia ejercieron en la distribución potencial para el modelo de la variedad pseudostrobus son la estacionalidad de la temperatura (Bio4: 48,1\%) y la temperatura media del cuatrimestre más cálido (Bio10: 29,3\%), y para la variedad apulcensis, la temperatura media del mes más húmedo (Bio8: $46 \%$ ) y la precipitación del cuatrimestre más frío (Bio19: 23,9 \%). Los modelos (M2 y M7) aportaron información valiosa sobre la distribución potencial y áreas óptimas para el desarrollo de ambas variedades, las cuales se encontraron en el Eje Neovolcánico y Sierra Madre del Sur, donde se pueden establecer UPGF para mejorar la calidad y producción de la semilla y la protección in situ de su acervo genético.

Los resultados generados permiten reducir los tiempos y costos para la selección de los mejores individuos para ser incluidos en programas de mejoramiento genético en Michoacán y Oaxaca. También se pueden diseñar planes integrales en los que se incluyan estrategias de manejo, conservación y restauración para garantizar la distribución continua y amplia del germoplasma de ambas variedades.

\section{AGRADECIMIENTOS}

Al Programa de Doctorado en Ciencias con Orientación en Manejo de Recursos Naturales de la Universidad Autónoma de Nuevo León, Facultad de Ciencias Foresta- 
les. Al Consejo Nacional de Ciencia y Tecnología (CONA$\mathrm{CyT}$ ) por el apoyo de beca de posgrado al primer autor. Al Sistema Biométrico Forestal por los datos proporcionados, y a los investigadores H. Jesús Muñoz Flores y Martin Gómez Cárdenas del Campo Experimental, Uruapan, Michoacán del INIFAP, por todas las facilidades brindadas durante la realización de este trabajo.

\section{REFERENCIAS}

Aguirre Gutiérrez J, HM Serna Chávez, AR Villalobos Arámbula, JA Pérez de la Rosa, N Raes. 2015. Similar but not equivalent: ecological niche comparison across closely-related Mexican white pines. Diversity and Distributions 21(3): 245-257. DOI: https://doi.org/10.1111/ddi.12268

Castellanos Acuña D, KW Vance Borland, JB St. Clair, A Hamann, J López Upton, E Gómez Pineda, JM Ortega-Rodríguez, C Saenz Romero. 2018. Climate-based seed zones for Mexico: guiding reforestation under observed and projected climate change. New forests 49(3): 297-309. DOI: https://doi.org/10.1007/s11056-017-9620-6

CONAFOR (Comisión Nacional Forestal, MX). 2019. Memoria Documental. Inventario Nacional Forestal y de Suelos. Zapopan, Jalisco, México. Consultado 18 de sep. 2019. Disponible en http://www.conafor.gob.mx:8080/documentos/ docs/8/4125CNF-24 Infys.pdf

Cuervo Robayo AP, O Téllez Valdés, MA Gómez Albores, CS Venegas Barrera, J Manjarrez, E Martínez Meyer. 2014. An update of high-resolution monthly climate surfaces for Mexico. International Journal of Climatology 34(7): 24272437. DOI: https://doi.org/10.1002/joc.3848

Cruz Cárdenas G, L López Mata, JT Silva, N Bernal Santana, F Estrada Godoy, JA López Sandoval. 2016. Potential distribution model of Pinaceae species under climate change scenarios in Michoacán. Revista Chapingo Serie Ciencias Forestales y del Ambiente 22(2): 135-148. DOI: https://doi. org/10.5154/r.rchscfa.2015.06.027

FAO (Organización de las Naciones Unidas para la Alimentación y la Agricultura, IT). 2018. El estado de los bosques del mundo-Las vías forestales hacia el desarrollo sostenible. Roma, Italia. FAO. Licencia: CC BY-NC-SA 3.0 IGO. 153 p.

Farjon A, BT Styles. 1997. Pinus (Pinaceae). Flora Neotropica. Monograph 75. New York, USA. The New York Botanical Garden. 291 p.

Flores A, J López Upton, CD Rallan Silva, AE Olthoff, R Alía, C Sáenz Romero, JM García del Barrio. 2019. Priorities for conservation and sustainable use of forest genetic resources in four Mexican pines. Forests 10(8): 675. DOI: https://doi. org/10.3390/f10080675

García Aranda MA, J Méndez González, JY Hernández Arizmendi. 2018. Distribución potencial de Pinus cembroides, Pinus nelsonii y Pinus culminicola en el Noreste de México. Ecosistemas y Recursos Agropecuarios 5(13): 3-13. DOI: https://doi.org/10.19136/era.a5n13.1396

GBIF (Global Biodiversity Information Facility). 2019. Bases de datos geográficos disponibles para Pinus pseudostrobus Lindl. var. pseudostrobus y Pinus pseudostrobus var. apulcensis Martínez en México. Global Biodiversity Information Facility. Consultado 15 de ago. 2019. Disponible en https://www.gbif.org/search?q=Pinus \%20pseudostro- bus\%20var\%20apulcensis

INEGI (Instituto Nacional de Estadística y Geografía, MX). 2019. Modelos Digitales de Elevación de Alta Resolución LiDAR. Edición 2012. México. Consultado 17 de ago. 2019. Disponible en https://www.inegi.org.mx/app/buscador/default.html?q=modelo+digital + de + elevacion

INEGI (Instituto Nacional de Estadística y Geografía, MX). 2008. Conjunto de datos vectoriales unidades climáticas. Edición 2008. México. Consultado 19 de ago. 2019. Disponible en https://www.inegi.org.mx/temas/climatologia/ default.html\#Descargas

Mantyka-Pringle CS, TG Martin, JR Rhodes. 2012. Interactions between climate and habitat loss effects on biodiversity: a systematic review and meta-analysis. Global Change Biology 18(4): 1239-1252. DOI: https://doi.org/13652486.2011.02593.x

Manzanilla Quiñones U, P Delgado Valerio, J Hernández Ramos, A Molina Sánchez, JJ García Magaña, M del C Rocha Granados. 2019. Similaridad del nicho ecológico de Pinus montezumae y P. pseudostrobus (Pinaceae) en México: implicaciones para la selección de áreas productoras de semillas y de conservación. Acta Botánica Mexicana 126: e1398. DOI: https://doi.org/10.21829/abm126.2019.1398

Martínez Méndez N, E Aguirre Planter, LE Eguiarte, JP Jaramillo Correa. 2016. Modelado de nicho ecológico de las especies del género Abies (Pinaceae) en México: Algunas implicaciones taxonómicas y para la conservación. Botanical Sciences 94(1): 5-24. DOI: https://doi.org/10.17129/ $\underline{\text { botsci. } 508}$

Osorio Olvera L, B Vijay, B Narayani, J Soberón, M Falconi. 2016. NicheToolbox: a web tool for exploratory data analysis and niche modeling. version 0.2.5.4. Consultado 25 de ago. 2019. Disponible en http://shiny.conabio.gob. $\underline{\mathrm{mx}: 3838 / \text { nichetoolb2/ }}$

Perret DL, AB Leslie, DF Sax. 2019. Naturalized distributions show that climatic disequilibrium is structured by niche size in pines (Pinus L.). Global Ecology and Biogeography 28(4): 429-441. DOI: https://doi.org/10.1111/geb.12862

Perry P J. 1991. The Pines of Mexico and Central America. Timber Press. Portland, Oregon. 231 p.

Peterson AT, J Soberon, RG Pearson, RP Anderson, E MartínezMeyer, M Nakamura, MB Araújo. 2011. Ecological niches and geographic distributions (MPB-49) (Vol. 56). Princeton University Press, Princeton, New Jersey 08540. 281 p.

QGIS Development Team. 2019. QGIS Geographic Information System. Open Source Geospatial Foundation. Consultado 10 de ago. 2019. Disponible en https://qgis.org/es/site/

Radosavljevic A, RP Anderson. 2014. Making better MaxEnt models of species distributions: complexity, overfitting and evaluation. Journal of Biogeography 41(4): 629-643. DOI: https://doi.org/10.1111/jbi.12227

Reynoso Santos R, MJ Pérez Hernández, W López Báez, J Hernández Ramos, HJ Muñoz Flores, JV Cob Uicab, MD Reynoso Santos. 2018. El nicho ecológico como herramienta para predecir áreas potenciales de dos especies de pino. $R e$ vista Mexicana de Ciencias Forestales 9(48): 47-68. DOI: https://doi.org/10.29298/rmcf.v8i48.114

Sáenz Romero C, GE Rehfeldt, NL Crookston, P Duval, J Beaulieu. 2009. Estimaciones de cambio climático para Michoacán: Implicaciones para el sector agropecuario y forestal y para la conservación de la Mariposa Monarca. Serie 3, 
Núm. 28. 21 p.

Secretaría de Economía. 2016. Declaratoria de vigencia de la Norma Mexicana: Establecimiento de unidades productoras y manejo de germoplasma forestal especificaciones técnicas. NMX-AA-169-SCFI-2016. Diario Oficial de la Federación. Consultado 15 de ago. 2019. Disponible en http://www.economia-nmx.gob.mx/normas/nmx/2010/ nmx-aa-169-scfi-2016.pdf

Singh H, RD Garg, HC Karnatak, A Roy. 2018. Spatial landscape model to characterize biological diversity using $\mathrm{R}$ statistical computing environment. Journal of Environmental Management 206, 1211-1223. DOI: https://doi.org/10.1016/j. jenvman.2017.09.055

Soberón JM. 2010. Niche and area of distribution modeling: a population ecology perspective. Ecography 33(1): 159-
167. DOI: https://doi.org/1600-0587.2009.06074.x

UNAM (Universidad Nacional Autónoma de México, MX). 2019. Herbario Nacional MEXU del Instituto de Biología. Universidad Nacional Autónoma de México. Consultado 10 de ago. 2019. Disponible en http://datosabiertos.unam. mx/IBUNAM:MEXU:104608

Viveros Viveros H, C Sáenz Romero, JJ Vargas Hernández, J López Upton. 2006. Variación entre procedencias de Pinus pseudostrobus establecidas en dos sitios en Michoacán, México. Revista Fitotecnia Mexicana 29(2): 121-126.

Worldclim. 2019. Worldclim versión 2.1. Datos climáticos históricos. Consultado 10 de sep. 2019. Disponible en https:// www.worldclim.org/data/worldclim21.html

Zobel BJ, JT Talbert. 1988. Técnicas de Mejoramiento Genético de Árboles Forestales. México D.F., México. Limusa. 545 p.

Recibido: 14/04/20

Aceptado: 23/06/20 
